\title{
A Distributed Method to Localization for Mobile Sensor Networks based on the convex hull
}

\author{
Yassine SABRI \\ STIC Laboratory \\ Chouaib Doukkali University, B.P: 20 \\ El Jadida MOROCCO
}

\author{
Najib EL KAMOUN \\ STIC Laboratory \\ Chouaib Doukkali University, B.P: 20 \\ El Jadida MOROCCO
}

\begin{abstract}
There has been recently a trend of exploiting the heterogeneity in WSNs and the mobility of either the sensor nodes or the sink nodes to facilitate data dissemination in WSNs. Recently, there has been much focus on mobile sensor networks, and we have even seen the development of small-profile sensing devices that are able to control their own movement. Although it has been shown that mobility alleviates several issues relating to sensor network coverage and connectivity, many challenges remain. Among these, the need for position estimation is perhaps the most important. Not only is localization required to understand sensor data in a spatial context, but also for navigation, a key feature of mobile sensors. This paper concerns the localization problem in the case where all nodes in the network (anchors and others sensors) are mobile. We propose the technique following the capabilities of nodes. Thus, each node obtains either an exact position or an approximate position with the knowledge of the maximal error born. Also, we adapt the periods where nodes invoke their localization. Simulation results show the performances of our method in term of accuracy and determinate the technique the more adapted related to the network configurations.
\end{abstract}

Keywords- wireless sensor network (WSN); Mobility; Localization; scalability.

\section{INTRODUCTION}

A wireless sensor network is composed of a large number of small and inexpensive smart sensors for many monitoring, surveillance and control applications. Each sensor makes its own local observation. All active sensors in the network coordinate to provide a global view of the monitored area. It is anticipated that such a network can be used in unattended environments or hostile physical locations. Applications include habitat monitoring [1][2], infrastructure surveillance [3], target tracking in tactical environments [4], etc. Almost all these applications require sensors to be aware of their physical locations. For example, the physical positions should be reported together with the corresponding observations in wildlife tracking, weather monitoring, location-based authentication, etc [5][6][7]. Location information can also be used to facilitate network functions such as packet routing [8][9] and collaborative signal processing [10], in which the complexity and processing overhead can be substantially reduced. Further, each node can be uniquely identified with its position, thus exempting the difficulty of assigning a unique ID before deployment [11]. However, many challenges exist in designing effective and efficient sensor self-positioning schemes for sensor networks. First, a localization algorithm must scale well to large sensor networks. Further, the location discovery scheme should not aggravate the communication and computation overheads of the network, since low-cost sensors have limited resource budgets such as battery supply, CPU, memory, etc. What's more, the localization scheme should not raise the construction cost of sensor nodes. Finally, the positioning scheme should be robust enough to provide high precision even under noisy environments.

This paper deals with the problem of localization in wireless sensor networks when sensors are mobile. There are three scenarios of mobility: sensors and anchors are mobile; sensors are mobile and anchors are static; sensors are static and anchors are mobile. For the last case, some methods have been proposed [12], [13]. In these methods, mobile anchors can be robots, humains, or other, equipped GPS which are used in order to locate others static sensors. In this paper, we present a new method to resolve the localization problem in the complex scenario where nodes and anchors are mobile. However, this method can be used for the two others cases of mobility. Three schemes are proposed following the capabilities of sensors. Sensors can be equipped with techniques like ToA/TdoA (Time of arrival / Time difference of arrival) or RSSI (Received Signal Strength Indicator) allowing computing distance between a pair of neighbor sensors. They may also be equipped with AoA (Angle of arrival) technique allowing computing angle between a pair of neighbor sensors. Finally, sensors may be equipped by none of these techniques. Our method determines an exact position for a sensor when it has at least two anchors in its neighborhood. Otherwise, it gives an approximate position and can compute in this case the generated maximal error. The localization problem with mobile sensors introduces a new problem: in fact, the energy of sensors being weak, each node cannot compute continually its localization in order to maintain accuracy position during its move.

Therefore, the question is: when a node must evoke the calculation of its position? In [14], authors compare three methods Static Fixed Rate (SFR), Dynamic Velocity Monotonic (DVM) and Mobility Aware Dead Reckoning Driven (MADRD). These methods define periods during which sensors should invoke their localizations. However, the authors assume that when a node invokes its position it obtains an exact localization (e.g. all sensors are equipped with GPS). These methods are explained in section II. However, when only a small number of sensors are anchors, the problem is not addressed. In this paper, we consider this case of network. 
When a node invokes its localization it does not always obtains its exact position: either it obtains an approximate position or it cannot locate itself. To overcome this problem, our method defines the periods when a node has to invoke its location. Finally, through simulations, we analyze performances of our three techniques.

The rest of the paper is organized as follows: In Section 2, we summarize related work on localization algorithms. In Section 3, introduces basic notions for this problem. In Section 4 and 5, we present our new localization algorithm. In Section 6 , we evaluate the proposed scheme through comprehensive simulation studies. We conclude the paper in Section 6.

\section{RELATED WORK AND BACKGROUND}

First, The popular Global Positioning System (GPS) [15] localization system may not be a practical solution for outdoor sensor networks. It is infeasible to install GPS on each sensor due to cost, form factors, power consumption and antenna requirements. Further, GPS requires direct Light-Of-Sight (LoS) communication, which renders it unfeasible for many outdoor application environments. Therefore in the past several years, extensive research has been directed to designing GPS-less location discovery schemes [16], [17], [18], [19], [20], [21], [22], [23], [24], [25], [26], [27], [28], [29], [30], [31], [32], [33], [34], [7], [35], [36] .These positioning algorithms differ in their assumptions on network deployment, device capabilities, node mobility, signal propagation, error requirement, etc. Thus, they can be classified differently.

For example, some methods are designed for static sensor networks, where sensors remain stationary after deployment, while others are for dynamic sensor networks where sensors and beacons are mobile [40]. These localization schemes can also be classified as centralized [37], [33], [39], where all computations are performed by a central point (e.g., the base station), or distributed, where sensors estimate their positions independently of each other. Centralized methods have poor scalability and are thus infeasible for large sensor networks. In this section, we will focus on distributed location discovery schemes for stationary sensor networks, which can be further classified as beacon-based and beacon-less depending on whether or not beacons are used, or classified as range-based and range-free according to the type of knowledge used in position estimation.

The majority of current location detection systems assume the existence of beacons, whose positions are known through GPS receivers or manual configuration. A typical sensor first measures the distances or angles from it to several beacons, and then obtains position estimation through techniques such as triangulation, trilateration, multilateration, etc. Based on the coverage capabilities of beacons, these localization systems can be further classified as systems with long-range beacons or systems with short-range beacons. Systems with long-range base stations have a fixed set of powerful beacons, whose transmission range can cover the entire network. Usually these base stations are manually deployed, are time-synchronized, and are equipped with special instruments such as directional antennas. In systems with shortrange beacons [33], [34], [7], [35], a small number of sensors with known positions are randomly deployed amongst other ordinary sensors. Some of them rely on transmitting both RF and ultrasound signals at the same time, where the RF is used for time-synchronizing the sender and the receiver.

Range-based localization relies on the availability of pointto-point distance or angle information. The distance/angle can be obtained by measuring Arrival (ToA), Time-Difference-ofArrival (TDOA), Received-Signal-Strength-Indicator (RSSI), and Angle-of-Arrival (AOA), etc. The range-based localization may produce fine-grained resolution, but have strict requirements on signal measurements and time synchronization. ToA measures the signal arrival times and calculates distances based on transmission times and speeds. GPS [16] is the most popular ToA-based localization system. By precisely synchronizing with a satellite's clock, GPS computes node position based on signal propagation time. Compared to ToA, TDoA has an advantage as the former's processing delays and non-LOS propagation can introduce larger errors [38]. Ref. [7] proposes a TDoA based scheme (AHLos) that requires base stations to transmit both ultrasound and RF signals simultaneously.

The RF signal is used for synchronization purposes. A sensor first measures the difference of the arrival times between the two signals, then determines the range to the base station. Finally, multilateration is applied to combine range estimates and generate location data. RSSI computes distance based on transmitted and received power levels, and a radio propagation model. RSSI is mainly used with RF signals [16], but the range estimation can be inaccurate due to multipath fading in outdoor environments [7]. AoA-based methods first measure the angle at which a signal arrives at a base station or a sensor, and then estimates the position using triangulation.

The calculation is quite simple, but AoA techniques require special antenna and may not perform well due to omnidirectional multipath reflections. Further, the signals can be difficult to measure accurately if a sensor is surrounded by scattering objects [12]. To the best of our knowledge, there is no other method, in the open literature that deals with this case. Some papers have been proposed in the case where anchors are mobiles and others sensors are static.

For example, [12], [13] uses robots or humans, which can be considered as anchors, move in the network and help others nodes to obtains their positions. When sensors are mobile, it is not reasonable that each sensor invokes its localization technique in order to locate itself continually, due to constraint of energy. A first work in [14] proposes three methods SFR (Static Fixed Rate), DVM (Dynamic Velocity Monotonic), MADRD (Mobility Aware Dead Reckoning Driven) to determinate periods where a node invokes its localization technique. But, it assumes that a node obtains its exact position when it invokes its localization (e.g. sensors are equipped with GPS). The following sub-sections explain these three methods.

A mobile sensor changes its position with time. A simple strategy for finding its position is the use of standard localization methods at any time. But if the position of the sensor is required frequently, this method is very costly. Tilak et. al tried to reduce the frequency of localizations for finding 
the position of sensors. They proposed techniques: Static Fixed Rate (SFR), dynamic Velocity Monotonic (DVM) and Mobility Aware Dead Reckoning Driven (MADRD). SFR calls a classical localization operation periodically with a fixed time interval. To respond a query from the base station, a sensor sends its position obtained from the last localization. When a sensor remains still or moves fast, in both cases, the reported position suffers a large error. In DVM, localization is called adaptively with the mobility of the sensors. The time interval for the next call for localization is calculated as the time required to traverse the threshold distance (a distance, traversed by the sensor, location estimation assumed to be error prone) with the velocity of the sensor between last two points in the sequence of localization calls. In case of high mobility, a sensor calls localization frequently. If a sensor suddenly moves with very high speed from rest, error in the estimated location becomes very high. In MADRD, the velocity is calculated from the information obtained from last two localized points.

The predictor estimates the position with this velocity and communicates to the query sender. At the localization point, the localized position is reported to the query sender and the distance error is calculated as the distance between the predicted position and reported position.

\section{PRELIMINARY}

Before In this paper, we focus on mobile sensor network. Moreover, we assume that all the sensors have identical transmission radius $r$. however, it is easy to adapt our method with sensors having different transmission radius. We represent a wireless sensor network as a graph $G(V, E)$ where

$V$ is the set of n nodes representing sensors and $\mathrm{E}$ is the set of $\mathrm{m}$ edges representing communication links. If two nodes $u$, $v$ are neighbors, then they are linked and the distance between $\mathrm{u}$ and $v$ is smaller than $r$. We assume also that some anchors have a priori knowledge of their own positions with respect to some global coordinate system (GPS) (black nodes in figures). We consider scenarios where nodes and anchors are mobile.

For example, in a military context, soldiers can be equipped with sensors and tanks with anchors. Soldiers use tank positions in order to obtain their positions. Finally, we should take into account functionalities of each sensors: for example, methods like RSSI or ToA/TDoA and AoA described in previous section. A wireless sensor networks is represented as a bidirectional graph $G(V, E)$ where $V$ is the set of $n$ nodes representing sensors and $E$ is the set of $m$ edges representing communication links. If two nodes $u, v \in V$ are neighbors, then they are linked that means distance between $u$ and $v$ is smaller than $r$. The set of neighbors for a node $u \in V$ is noted $N(u)$.Anchor nodes have knowledge of their location through some other means, such as GPS or simply explicit programming. The set of anchors is noted $\Lambda$. The set of neighbor anchors for a node $u$ is noted $N_{\Lambda}(u)\left(N_{\Lambda}(u)=N(u) \cap \Lambda\right)$ and the set of non-neighbor anchors is noted $\overline{N_{\Lambda}}(u)\left(\overline{N_{\Lambda}}(u)=\Lambda / N_{\Lambda}(u)\right)$. Note that all identical nodes (anchors or others nodes) have the same capabilities (energy, processing, communication, ...). The coordinate of a position of node $u$ is noted $\left(x_{u}, y_{u}\right) . \mathrm{P}$ is the set of all possible positions in a network. Our method construct the convex hull of a point cloud $\mathrm{S}_{u}$ for each node $u$ ,this convex hull is noted $\operatorname{conv}\left(\mathrm{S}_{u}\right)$. The localization modules (eg, GPS or Galileo) are expensive and consumers of energy,for this our method seeks to use the least possible anchors with the Nodes can use technology measures distances as ToA, RSSI, AoA. So, when it receives a signal from a transmitter, a node deduces that it is located on the circle centered on the transmitter. The exact distance between two nodes $u$ and $v$ is noted $d_{u v}$. Two neighbor nodes $u, v$ know $d_{u v}$ (via ToA, ...). The estimated distance is noted $\hat{d}_{u v}$. The following section explains how to obtain these estimated distance. the set of circles built from the knowledge of anchor neighbors is noted $\mathrm{C}_{N_{\Lambda}}$, the set of circles built from the knowledge of non-anchor neighbors is noted $\mathrm{C}_{\overline{N_{\Lambda}}} \cdot \varepsilon$ is the distance between the estimated position ${\left(x_{u_{e s t m}}, y_{u_{e s t m}}\right)}$ of the sensor $u$ and the summit furthest from convex hull $\operatorname{Conv}(\mathrm{S})$. Let $d_{\text {err }}$ being the distance between the estimated position of a node and its real position, representing the position error. The node knows that $d_{e r r}$. By using a predefined threshold, if $d_{\text {err }} \leq$ threshold then the node has an estimation close to its real position. In this case the node becomes an estimated anchor and broadcasts its position .

\section{LOCALIZATION TECHNIQUE}

\section{A. Localization Algorithm Based on the convex hull}

The Initially, each anchor broadcasts its position. A node can therefore be deduced the distance between each of the anchors We use the technique SumDist (Savvides et al., 2002) for estimating distances adding the distances between separated sensor nodes of an anchor.Upon receiving the position of a anchor, a node considers the following cases:

- If it receives directly the position of the anchor, he deduces they are neighbors and therefore it located on the circle centered at the anchor or radius of a circle is $r$.

- If it receives the position by an intermediate node, it concluded that it is not neighbor of the anchor and therefore it is not inside the circle of radius $r$ centered in anchor. So,when a node $u$ receives a position of an anchor $A$, it estimates the distance to this anchor with Sum-Dist and draws one or two circles. In fact, if $\left(A \in N_{\Lambda}(u)\right), u$ knows $d_{A u}$ and deduces that it is on the circle $\mathrm{C}_{A u}$ of radius equals to $d_{A u}$ and centered in $A$. If $\left(A \notin N_{\Lambda}(u)\right)$ then $u$ knows that it is not inside the circle of center $A$ and radius $r$ otherwise $A$ and $u$ would be neighbors. Moreover, $u$ knows the estimated distance to $A, \hat{d}_{A u}$ deduced by Sum-Dist. By triangular 
inequality, $\hat{d}_{A u} \leq d_{A u} \cdot u$ applies this technique to each received anchor position. So, $u$ is inside the circle $\mathrm{C}_{\overline{A u}}$ of center $A$ and radius $\hat{d}_{A u}$. Thus, the intersection of circles defines a cloud of points $S_{u}$. the center of gravity of the convex hull of this cloud $\operatorname{conv}\left(\mathrm{S}_{u}\right)$ represents the estimated position of $u$.

To summarize, for each node $u \in V / \Lambda$, the envelope obtained as follow:

Initialization of the algorithm:

$$
\begin{gathered}
\mathrm{S}_{0}=\mathrm{P} \\
\mathrm{C}_{N_{\Lambda}(u)_{0}}=\mathrm{C}_{\overline{N_{\Lambda}}(u)_{0}}=\{\varnothing\}
\end{gathered}
$$

When a node $u$ receives a message controle $P$ from anchor node $a_{i}$ neighbor:

$$
\text { If } a_{i} \in N_{\Lambda}(u) \text { : }
$$

The circle centered at $a_{i}$ and of radius $d_{u a_{i}}^{2}$ :

$$
\mathrm{C}_{u a_{i}}=\left\{\left(x_{i}, y_{i}\right) \in \mathrm{P} \mid\left(x_{i}-x_{a}\right)^{2}+\left(y_{i}-y_{a}\right)^{2}=d_{u a_{i}}\right\}
$$

Construction of intersection points of a circle $\mathrm{C}_{u a_{i}}$ with the old circles $\mathrm{C}_{(u)_{i-1}}$,keeping only the points inside a circle centered at $u$ and of radius $\varepsilon_{u_{i-1}}$ :

$\left.\mathrm{W}_{u_{i}}=\left\{\left(x_{i}, y_{i}\right) \in\left(\mathrm{C}_{(u)_{i-1}} \cap \mathrm{C}_{u a_{i}}\right) \mid\left(x_{i}-x_{u_{\text {essm }}}\right)_{i}\right)^{2}+\left(y_{i}-y_{u_{e s t m}}\right)^{2} \leq \varepsilon_{u_{i-1}}^{2}\right\}$

Cleaning the old cloud of points $S_{i-1}$, keeping only the points inside a circle centered at $u$ and of radius $d_{u a_{i}}$ :

$\mathrm{Z}_{u_{i}}=\left\{\left(x_{i}, y_{i}\right) \in \mathrm{S}_{i-1} \mid\left(x_{i}-x_{a}\right)^{2}+\left(y_{i}-y_{a}\right)^{2} \leq d_{u a_{i}}^{2}\right\}$

New cloud of points $S_{i}$ :

$$
\mathrm{S}_{i}=\mathrm{Z}_{u_{i}} \cup \mathrm{W}_{u_{i}}, i \geq 3
$$

The circle $\mathrm{C}_{u a_{i}}$ joins the old circles $\mathrm{C}_{N_{\Lambda}\left(u_{i-1}\right.}$ :

$$
\mathrm{C}_{N_{\Lambda}(u)_{i}}=\mathrm{C}_{u a_{i}} \cup \mathrm{C}_{N_{\Lambda}(u)_{i-1}}
$$

Same effect occurs when a node $u$ receives a message controle $P$ from anchor node $a_{i}$ not neighbor :

$$
\begin{aligned}
& \text { if } a_{i} \notin \overline{N_{\Lambda}}(u) \\
& \mathrm{C}_{\overline{u a_{i}}}=\left\{\left(x_{i}, y_{i}\right) \in \mathrm{P} \mid\left(x_{i}-x_{a}\right)^{2}+\left(y_{i}-y_{a}\right)^{2}=\hat{d}_{u a_{i}}^{2}\right\} \\
& \mathrm{W}_{u_{i}}=\left\{\left(x_{i}, y_{i}\right) \in\left(\mathrm{C}_{(u)_{i-1}} \cap \mathrm{C}_{\overline{u a_{i}}}\right) \mid\left(x_{i}-x_{u_{e s t m}}\right)^{2}+\left(y_{i}-y_{u_{e s t m}}\right)_{i}^{2} \leq \varepsilon_{u_{i}-1}^{2}\right\} \\
& \mathrm{Z}_{u_{i}}=\left\{\left(x_{i}, y_{i}\right) \in \mathrm{S}_{i-1} \mid r^{2} \leq\left(x_{i}-x_{a}\right)^{2}+\left(y_{i}-y_{a}\right)^{2} \leq \hat{d}_{u a_{i}}^{2}\right\} \\
& \mathrm{S}_{i}=\mathrm{Z}_{u_{i}} \cup \mathrm{W}_{u_{i}}, i \geq 3 \\
& \mathrm{C}_{\overline{N_{\Lambda}}(u)_{i}}=\mathrm{C}_{\overline{u a_{i}}} \cup \mathrm{C}_{\overline{N_{\Lambda}}(u)_{i-1}}
\end{aligned}
$$

$\mathrm{C}_{(u)_{i}}=\mathrm{C}_{N_{\Lambda}(u)_{i}} \cup \mathrm{C}_{\overline{N_{\Lambda}}(u)_{i}}$

The end for each node we will have a set of points $S_{u}$ of the cloud:

$$
\mathrm{S}_{u}=\left\{p_{1}, p_{2}, p_{3}, \cdots, p_{n}\right\}
$$

Calculate the convex hull $\mathrm{S}_{u}$ based on Jarvis' March:

$\operatorname{conv}\left(\mathrm{S}_{u}\right)=\left\{\sum_{n=0}^{n} \alpha_{i} p_{i} \mid \alpha_{i} \geq 0, \sum_{i} \alpha_{i}=1\right\}$

The new estimation error $\varepsilon_{u_{i}}$ :

$$
\varepsilon_{u_{i}}=\max _{p \in c o n \Downarrow S)} d\left(p, u_{e s t m_{i}}\right)
$$

The main design of the Slsnj, which is a simple finite state machine. As shown in figure 1, a node running Slsnj is in one of four states at any time: (i) Sensor not estimated, (ii) Sensor estimated, (iii) estimated Anchor,and (iv) improve the accuracy. Transitions between the states are triggered by events. After the Slsnj protocol is initiated, the node enters the Sensor not estimated state, Whenever the node receives a broadcasting ProbePacket packet, the node enters the Sensor not estimated state and uses this packet to estimate its postion,after this stage of estimation the node switches to another state is depending on the value of the estimation error found, if espilon<threshold the node enters in estimated Anchor state else it enters in Sensor estimated state .In the latter two states a node is still waiting of probpacket packet from anchor or estimated Anchor nodes to enter in improve the accuracy state and improve its accuracy.when there will be no more ProbePacket, the node switches to the state final and considered as estimated with an error of precison. An example is illustrated in figure 2. $X$ receives positions of anchors $A, B$ and $C$. It estimates distances $\hat{d}_{A X}, \hat{d}_{B X}, \hat{d}_{C X}$ with Sum-Dist. Since all an- chors are not neighbors of $X$ then $X$ is not inside circles centered respectively in $A, B, C$ with a radius equals to $r$ but it is inside circles with radius equal to $\hat{d}_{A X}, \hat{d}_{B X}, \hat{d}_{C X}$. The intersection of these circles defines the cloud points $\mathrm{S}_{X}$ for a node $X . X$ computes the center of gravity of the convex hull $\operatorname{conv}\left(\mathrm{S}_{X}\right)$ of this cloud and estimates its position in $G 2$.

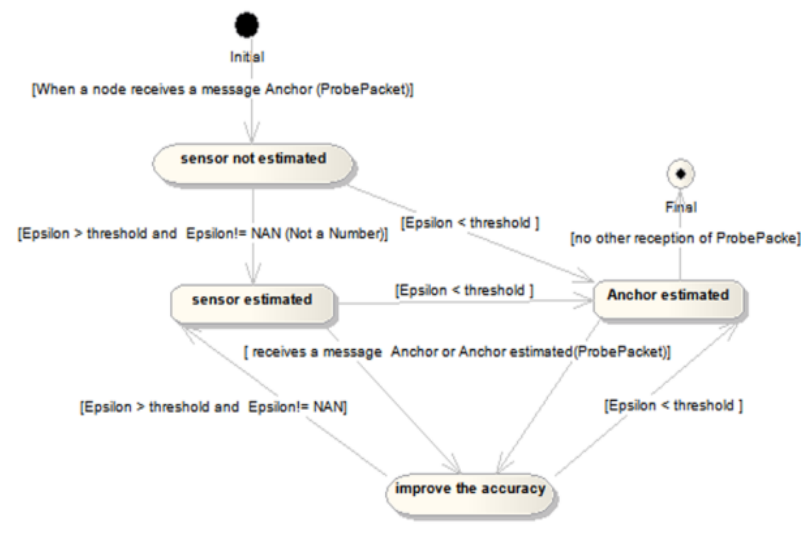

Figure 1: State machine diagram for Sensor node not estimated 


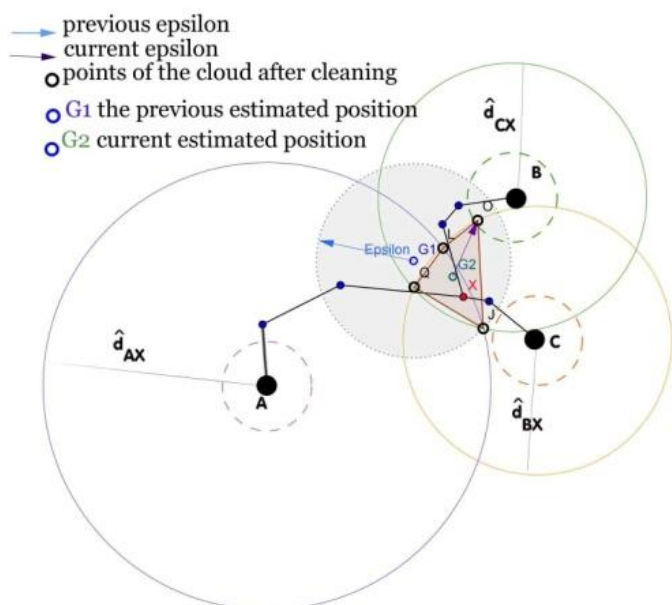

Figure 2: Example of estimating the position for $\mathrm{X}$ at the reception of localization information from $\mathrm{C}$ anchor

\section{B. Pseudo-code}

The pseudo-code for the Slsnj is shown in figure 3. Each anchor exact (equipped with GPS or Galileo) or estimated broadcasts its position through the control message $\mathrm{P}$, and depending on number of hops traveled by the packet $\mathrm{P}$ we check its validity,if the number of hops is less than a certain threshold called ThresholdHopcount it is considered confirmed otherwise a packet is rejected.after we apply our method as described previously .

\section{C. $P$ roperties}

Our localization technique meets three very important properties who have a significant impact on its performance:

- First, a node knows if its estimated position is close to its real position. Let $\varepsilon$ be the distance between the center of gravity and the point, in the zone, furthest away from the center of gravity. Let $d_{e r r}$ being the distance between the estimated position of a node and its real position, representing the position error. The node knows that $d_{e r r} \leq \varepsilon$.

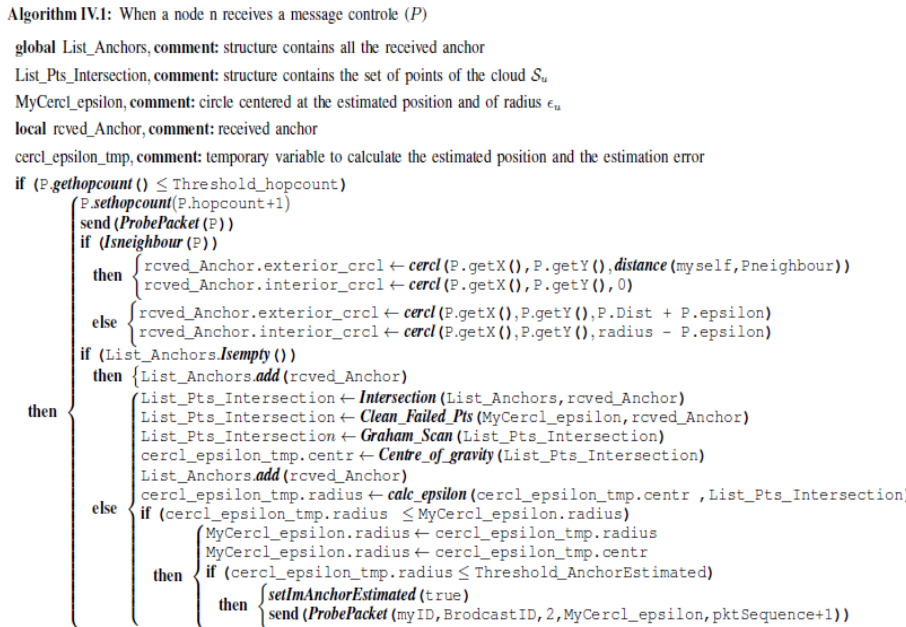

Figure 3: Description of algorithm Slsnj
By using a predefined threshold, if $\varepsilon \leq$ threshold then the node has an estimation close to its real position. In this case the node becomes an estimated anchor and broadcasts its position and its $\varepsilon$. When a node applies the approximation technique with an estimated anchor radius, it takes into account $\varepsilon$.Consider a sensor $X$ calculating its position with an estimated anchor $A$. If they are neighbors, $X$ trace two circles (belongs to $\mathrm{C}_{N_{\Lambda}}$ ) centered in $A$ of radius $d_{A X} \pm \varepsilon$ and deduce that it is between these two circles. If they are not neighbors, $X$ deduces that it is not inside the circles centered at $A$ of radius $r-\varepsilon$ and belongs to a circle of radius $d_{A X}+\varepsilon$,the definitions (4),(6),(9) and (11) become:

si $a_{i} \in N_{\Lambda}(u)$ :

$\mathrm{C}_{u a_{i}}=\left\{\left(x_{i}, y_{i}\right) \in \mathrm{P} \mid\left(x_{i}-x_{a}\right)^{2}+\left(y_{i}-y_{a}\right)^{2}=\left(d_{u a_{i}} \pm \varepsilon_{u_{i}}\right)^{2}\right\}$

$\mathbf{Z}_{u_{i}}=\left\{\left(x_{i}, y_{i}\right) \in \mathrm{S}_{i-1} \mid\left(x_{i}-x_{a}\right)^{2}+\left(y_{i}-y_{a}\right)^{2} \leq\left(d_{u a_{i}} \pm \varepsilon_{u_{i}}\right)^{2}\right\}$

si $a_{i} \notin \overline{N_{\Lambda}}(u)$ :

$\mathrm{C}_{\overline{u a_{i}}}=\left\{\left(x_{i}, y_{i}\right) \in \mathrm{P} \mid\left(x_{i}-x_{a}\right)^{2}+\left(y_{i}-y_{a}\right)^{2}=\left(\hat{d}_{u a_{i}} \pm \varepsilon\right)^{2}\right\}$

$\mathbf{Z}_{u_{i}}=\left\{\left(x_{i}, y_{i}\right) \in \mathrm{S}_{i-1} \mid(r-\varepsilon)^{2} \leq\left(x_{i}-x_{a}\right)^{2}+\left(y_{i}-y_{a}\right)^{2} \leq\left(\hat{d}_{u a_{i}}+\varepsilon\right)^{2}\right\}$

- Second, a node can detect if some informations are wrong. This case is illustrated in esxpresion $\mathrm{W}_{u_{i}}$.With its bound error $\varepsilon$, nodes reject the cloud points that are outside of circle centered at its estimated position and of radius $\varepsilon$.for example, when a node $u$ detects a point of its cloud $\mathrm{S}_{u}$ it outside in the circle centered at $u$ of radius $\varepsilon$ will not take it into account . This property is defined by the expression $\mathrm{W}_{u_{i}}$.

- Third, convex hull algorithms as Graham scan [?] and Jarvis march [?] allowed us to calculate the convex hull $\operatorname{conv}(\mathrm{S})$ a cloud of points with a very optimum complexity, of order $O(n \log (n))$ with $n$ the number of points of the cloud, which allowed us to reduce consumption of CPU time (and therefore energy), but also allowed us to optimize particularly the consumption of memory storage ,focusing not on global interpretation of the network as in an algorithm of type Grid-scan, but only on points of the cloud. The improvement made allowed us to retain the properties functional Our localization technique despite the change in network size, and efficiently localize the nodes (continuously) and with a certain level of quality in different scales.

\section{Structure of the control message exchanged}

Our approach Requires the exchange of Specific Information. For this, a specific control message is designed. The fields in this message, called ProbePacket, exchanged during the execution of the localization algorithm are shown in Table 1 ,tow possible values for the packet subject are used in the algorithm:Anchor,Anchor estimated. Note that when a node broadcasts or sends a message in a wireless network, all 
nodes in its scope communication receive this message. The validation of a control message is limited by a threshold of validation,called Threshold_hopcount.

\begin{tabular}{|r|l|}
\hline Srcld & $\begin{array}{l}\text { global identifier ID of the transmitter node of } \\
\text { the packet }\end{array}$ \\
\hline Destld & $\begin{array}{l}\text { global identifier ID the receiving node } \\
\text { type of the transmitter node of the packet (An- } \\
\text { chor, Anchor estimated) }\end{array}$ \\
\hline MyCerc_epsilon & $\begin{array}{l}\text { circle centered at the estimated position or exact } \\
\text { position of the transmitter, and of radius } \epsilon(0 \\
\text { for transmitter Anchor ) }\end{array}$ \\
\hline Hopcount & $\begin{array}{l}\text { the number of hops between the anchor trans- } \\
\text { mitter and the sensor receiver }\end{array}$ \\
\hline pktSequence & $\begin{array}{l}\text { to avoid the redundantly reception of the same } \\
\text { message }\end{array}$ \\
\hline Dist & $\begin{array}{l}\text { the sum of the distances intermediate between } \\
\text { the anchor transmitter and the receiver sensor }\end{array}$ \\
\hline
\end{tabular}

Figure 4: Fields of the message ProbePacket

\section{ADAPTATION OF DVM AND MADRD}

DVM and MADRD determine periods when a node has to invoke its localization technique, related to mobility of nodes. It is necessary to adapt these two techniques in order to take into account accuracy of localization. SFR is not concerned by this problem because its period of time is constant. In these techniques, when a node is moving fast, localization will be carried out more often and conversely. But if a node is located with important error, it is necessary to invoke localization technique more often. Therefore, if node is located with high accuracy,methods DVM and MADRD do not need any change but if node obtains an approximate position then protocols DVM and MADRD have to take into account the error $\varepsilon$.Let

$t$ be the time returned by DVM or MADRD and $t$ the time returned by our method when $\varepsilon$ is taken into account.If $\varepsilon=0$ (ie. the position is exact)then $t=t^{\prime}$ and if $\varepsilon \geq r$ (ie.the position is bad then $t^{\prime}=0$.

Between these two values, $t^{\prime}$ varies linearly $t^{\prime}=t-\varepsilon \frac{t}{r}$ .Thus, If $\varepsilon$ represents an important error,then periods during which a node should invokes its localization will be short and conversely if $\varepsilon$ is a small error. Perturbation of predictions in MADRD : In MADRD nodes calculate their positions related to predictions.A node computes its position related to its previous position. conversely if a small error. Perturbation of predictions in MADRD : In MADRD nodes calculate their positions related to predictions. A node computes its position related to its previous position.

\section{SIMULATIONS}

\section{A. Environnement de simulation}

Experiments were built upon the J-Sim simulator [9] dedicated to WSN simulations. It is a compositional, component based simulation environment. It is built upon the concept of autonomous component programming model. JSim is developed entirely in Java. The signal attenuation due to obstacles or other factors (e.g. use of unidirectional antennas) is simulated in J-Sim. Therefore, the vicinity of a node in terms of transmission range is not necessarily spherical. Note that there several simulators in the literature such as GlomoSim[41], OMNET++[42], OPNET[43] , NS-
2[44]. The MAC layer is considered perfect and the transmission of messages are without loss in our simulation.

In order to allow easy comparison between different scenarios, range errors as well as errors on estimated positions are normalized to the radio range. For example, of position error means a distance of half the range of the radio between the real and estimated positions. The percentage of range errors is noted.

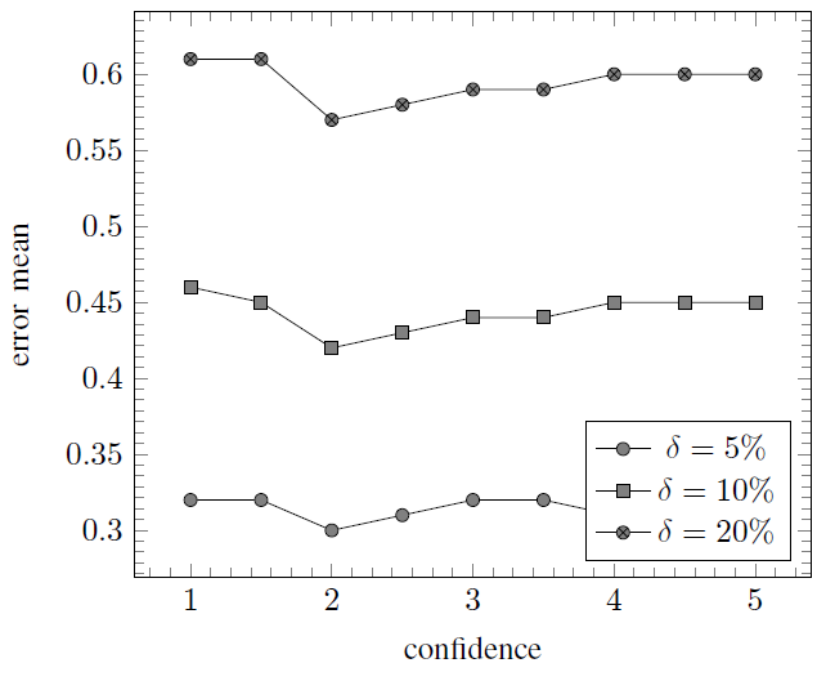

Figure 5: impact of threshold confidence

\section{B. Results}

Globally, the positions determined by a localization algorithm represent a geometrical layout of the physical positions of the sensors. This layout must be compared to the ground truth, or known layout of the sensors. It is important therefore that not only the error between the estimated and real position of each node is minimized, but also that the geometric layout determined by the algorithm matches well the original geometric layout. In order to have a unified approach for evaluate the accuracy of our technique and a solid frame for analysis of the scalability, we propose to use two metrics.

- MAE(Mean Absolute Error): The simplest way to describe localization performance is to determine the residual error between the estimated and actual node positions for every node in the network, sum them and average the result. Broxton et al in [45] do this using the mean absolute error metric (MAE), which, for each of $\mathrm{n}$ nodes in the network, calculates the residual between the estimated nodes and actual coordinates.

$M A E=\frac{\sum_{i=1}^{n}\left(x_{i}-\hat{x}_{i}\right)^{2}-\left(y_{i}-\hat{y}_{i}\right)^{2}}{n}$

with $\left(x_{i}, y_{i}\right)$ the real position and $\left(\hat{x}_{i}, \hat{y}_{i}\right)$ the estimated postilions .

- GDE (Global Distance Error): As discussed briefly at the start, it is important for the accuracy metric to reflect not only the positional error in terms of distance, but also in terms of the geometry of the network localization result. GDE in 
[46] takes the RMS error over the network of $\mathrm{n}$ nodes and normalises it using the constant $\mathrm{R}$. In Ahmed et als context, $\mathrm{R}$ represents average radio range, meaning the localization results are represented as a percentage of the average distance nodes can communicate over.

$G D E=\frac{1}{r} \sqrt{\frac{\sum_{i=1}^{n} \sum_{j=i+1}^{n}\left(\frac{\hat{d}_{i j}-d_{i j}}{d_{i j}}\right)^{2}}{n(n-1) / 2}}$

with $\hat{d}_{i j}$ The estimated distance between $i$ and $j$ and $d_{i j}$ The actual distance between $i$ and $j$.

This section analyses the performances of our three methods related to the techniques SFR, DVM and MADRD. Mobility model: The mobility model used in this paper is the random waypoint model [47]. It is the classical model used in the mobile network. In this model, velocities of nodes vary and a node can stop its move. Each node picks a random location and starts moving to it. As soon as the node reaches the destination, it picks another destination randomly and moves toward it. Our simulation uses the BonnMotion tool to generate the various scenarios of mobility where velocity and trajectory deviation of nodes vary. Each scenario runs during 90 seconds.

Simulation model: In our simulations, all messages are delivered. For easier comparison between different scenarios, range errors as well as estimations of position errors are normalized to the radio range. This technique is classical in the literature and allows comparisons with others methods. For example, of position error means a distance equal to half of the radio range between the real and estimated positions. In our scenarios, we use nodes in a square of . The transmission range of nodes is equal to . Among nodes, we randomly select anchors with representing a density of anchors in the square from to . Also, we consider measure errors of , and respectively. Analyse: In our method, it is possible that a node does not obtain an estimated position when it does not contain anchors in its neighborhood. This case depends on the anchors density. Therefore, if our simulations consider only the position average error rate of sensors, performances of our three techniques would not be shown due to this case. As a consequence, our results focus on the time during which a node is located with a position error lower than for MAE metric and for GDE metric. After this time, nodes are considered that they are badly positioned. For our analysis, we perform tests. For each scenario, we take into account the mean and we represent on graphs the confidence interval. Here, there is of chance that the real values belong to this interval.

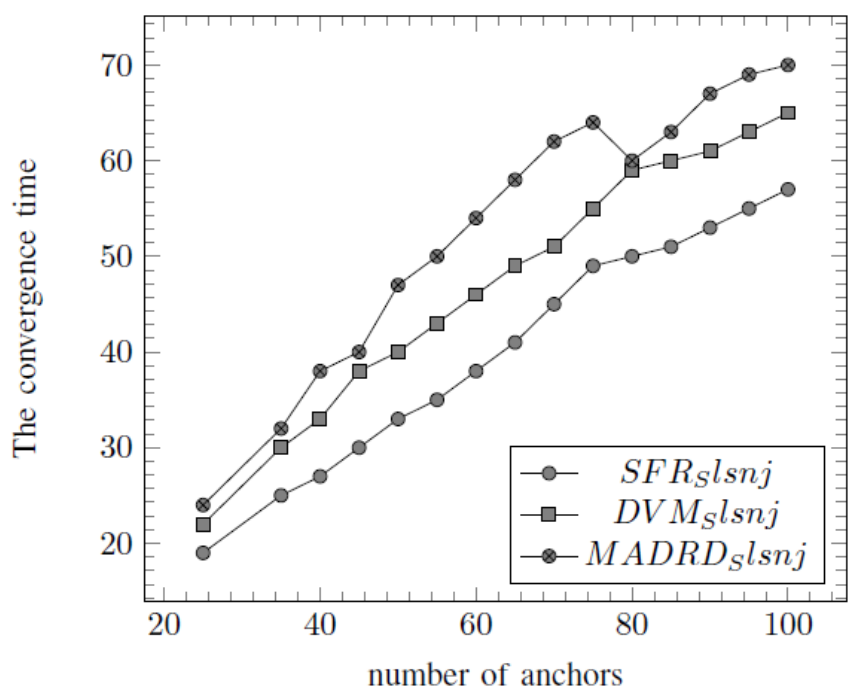

Figure 6: GDE Performances of SFR, DVM and MADRD with errors equal

to $5 \%$

1) Without measure errors : In this section, we consider the ideal case where measure errors are equal to. The figures 7 and 9 show simulations with SFR, DVM and MADRD for GDE and MAE. These curves represent the time during which a node is located with a position error lower than for MAE for GDE. For example, in figures 5, when the network contains 70 anchors, a node is located with an error lower than during: 41.01s. Without surprise, accuracy of positions is based on the capability of nodes to calculate distances. MADRD provides better results than DVM and then SFR.

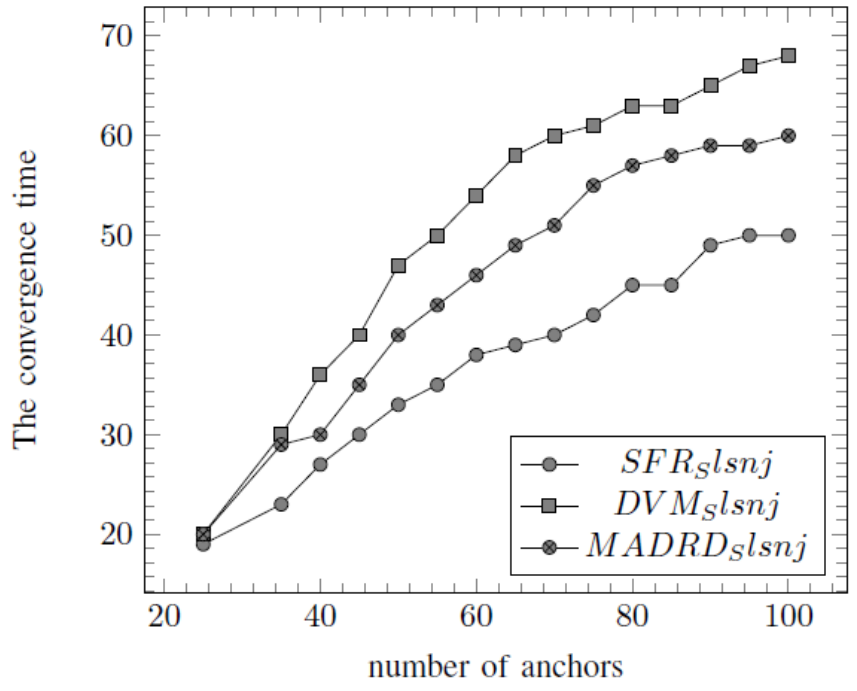

Figure 7: GDE Performances of SFR, DVM and MADRD without measure

Errors 
2) Measure errors equal to $5 \%:$ In this section, we introduce measure errors equal to 5\%. Figure 6 and 8 shows that results obtained when nodes can calculate distances, is not influenced too much by measure errors in SFR, DVM and MADRD for MAE and GDE. To conclude, DVM provides better results than MADRD and then SFR.

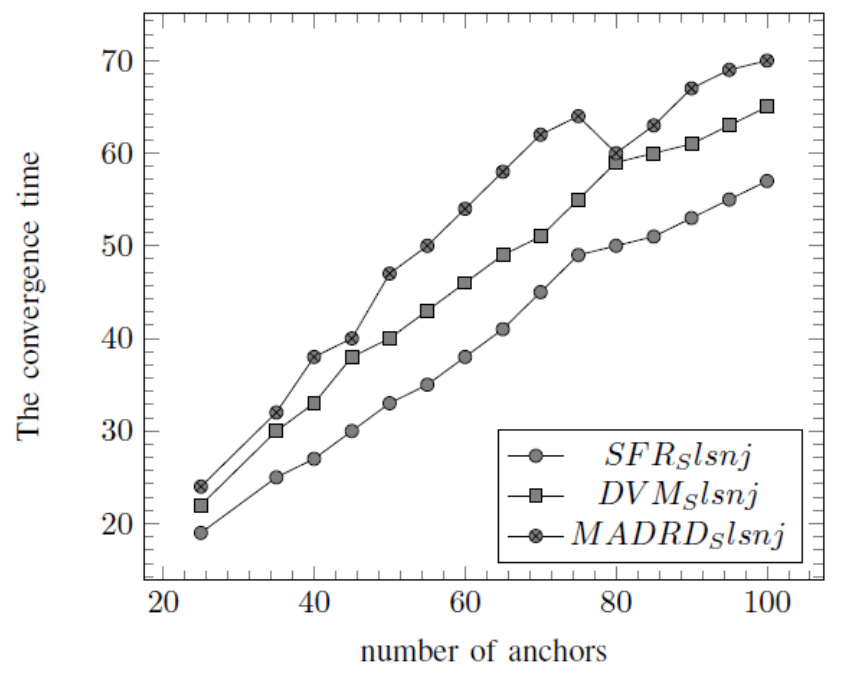

Figure 8: MAE Performances of SFR, DVM and MADRD with errors equal

$$
\text { to } 5 \%
$$

3) Conclusions of simulations : In These simulations show the performances of our method and show how to adapt SFR, DVM and MADRD, related to the network environment in order to provide good results. We note the impact of measure errors in MADRD since it is efficient only if it uses accurate positions. MADRD provides good results in a network environment without measure errors, but when we introduce errors, DVM is the best. Finally, phenomenons seen in an environment with measure errors equal to 5 errors equal to 10 .

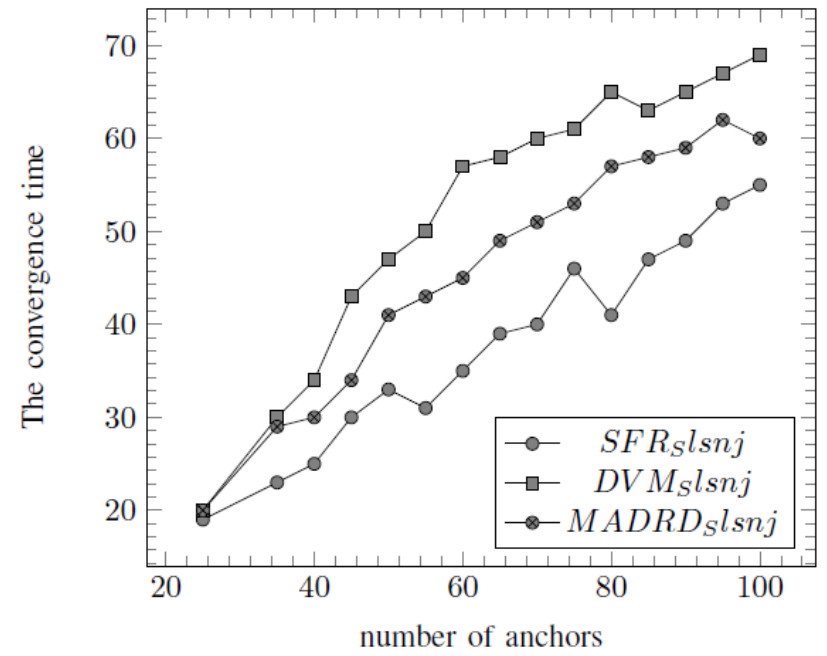

Figure 9: MAE Performances of SFR, DVM and MADRD without measure

errors

\section{CONCLUSIONS}

This paper proposes the method for the localization problem when anchors and others sensors are mobile. this method take into account capabilities of nodes: nodes which can calculate either distances with their neighbors . Moreover, in order to answer to question when a node should invoke its position? related to network environment and capabilities of nodes, we adapted techniques SFR, DVM, MADRD, proposed in [14]. Our simulations show the performances of our method and determinate the technique the more adapted related to the network configurations.

\section{REFERENCES}

[1] Cerpa, J. Elson, M. Hamilton, J. Zhao, D. Estrin, and communications technology," in Workshop on Data communication in Latin America and the Caribbean, ser. SIGCOMM LA '01. New York, NY, USA: ACM, 2001, pp. 20-41.

[2] A. Mainwaring, D. Culler, J. Polastre, R. Szewczyk, and J. Anderson,"Wireless sensor networks for habitat monitoring," in Proceedings of the 1st ACM international workshop on Wireless sensor networks and applications, ser. WSNA '02. New York, NY, USA: ACM, 2002, pp. 88-97.

[3] T. van Dam and K. Langendoen, "An adaptive energy-efficient mac protocol for wireless sensor networks," in Proceedings of the $1^{\text {st }}$ international conference on Embedded networked sensor systems, ser. SenSys '03. New York, NY, USA: ACM, 2003, pp. 171-180. [Online]. Available : http ://doi.acm.org/10.1145/958491.958512

[4] Q. Fang, F. Zhao, and L. Guibas, "Lightweight sensing and communication protocols for target enumeration and aggregation," in Proceedings of the 4th ACM international symposium on Mobile ad hoc networking \& computing, ser. MobiHoc '03. New York, NY, USA : ACM, 2003, pp. 165-176.

[5] C. Intanagonwiwat, R. Govindan, D. Estrin, J. Heidemann, and F. Silva, "Directed diffusion for wireless sensor networking," IEEE/ACM Trans. Netw., vol. 11, no. 1, pp. 2-16, Feb. 2003. [Online]. Available : http ://dx.doi.org/10.1109/TNET.2002.808417

[6] S. Madden, M. J. Franklin, J. M. Hellerstein, and W. Hong, "Tag: a tiny aggregation service for ad-hoc sensor networks," SIGOPS Oper. Syst. Rev., vol. 36, no. SI, pp. 131-146, Dec. 2002.

[7] A. Savvides, C.-C. Han, and M. B. Strivastava, "Dynamic fine-grained localization in ad-hoc networks of sensors," in Proceedings of the $7^{\text {th }}$ annual international conference on Mobile computing and networking, ser. MobiCom '01. New York, NY, USA : ACM, 2001, pp. 166-179.

[8] S. De, C. Qiao, and H. Wu, "Meshed multipath routing with selective forwarding: an efficient strategy in wireless sensor networks," Computer Networks, vol. 43, no. 4, pp. 481-497, 2003.

[9] J. Li, J. Jannotti, D. S. J. De Couto, D. R. Karger, and R. Morris, “A scalable location service for geographic ad hoc L. Girod, "Habitat monitoring: application driver for wireless For papers published in translation journals, please give the English citation first, followed by the original foreign-language citation routing," in Proceedings of the 6th annual international conference on Mobile computing and networking, ser. MobiCom '00. New York, NY, USA: ACM, 2000, pp. 120-130.

[10] J. Heidemann and N. Bulusu, "Using geospatial information in sensor networks," 2001.

[11] C. Schurgers, G. Kulkarni, and M. B. Srivastava, "Distributed ondemand address assignment in wireless sensor networks," IEEE Trans. Parallel Distrib. Syst., vol. 13, no. 10, pp. 1056-1065, Oct. 2002.

[12] N. B. Priyantha, H. Balakrishnan, E. Demaine, and S. Teller, "MobileAssisted Localization in Wireless Sensor Networks," in IEEE INFOCOM, Miami, FL, March 2005.

[13] N. Bulusu, J. Heidemann, and D. Estrin, “Adaptive beacon placement," 2001, pp. 489-498. 
[14] S. Tilak, V. Kolar, N. B. Abu-Ghazaleh, and K.-D. Kang, "Dynamic localization protocols for mobile sensor networks," CoRR, vol. cs.NI/0408042, 2004.

[15] B. Hofmann-Wellenhof, H. Lichtenegger, and J. Collins, Global Positioning System: Theory and Practice, 5th ed. Springer, Feb. 2001.

[16] P. Bahl and V. N. Padmanabhan, "RADAR: an in-building RF-based user location and tracking system," INFOCOM 2000. Nineteenth Annual Joint Conference of the IEEE Computer and Communications Societies. Proceedings. IEEE, vol. 2, pp. 775-784 vol.2, 2000.

[17] N. Bulusu, J. Heidemann, and D. Estrin, "Gps-less low cost outdoor localization for very small devices," IEEE Personal Communications Magazine, vol. 7, no. 5, pp. 28-34, October 2000.

[18] "Adaptive beacon placement," in Proceedings of the The $21^{\text {st }}$ International Conference on Distributed Computing Systems, ser. ICDCS '01. Washington, DC, USA: IEEE Computer Society, 2001, pp. 489-.

[19] S. Capkun and J.-P. Hubaux, "Secure positioning of wireless devices with application to sensor networks," in INFOCOM, 2005, pp. 1917- 1928.

[20] X. Cheng, A. Thaeler, G. Xue, and D. Chen, "Tps: A time-based positioning scheme for outdoor wireless sensor networks," in IEEE INFOCOM, 2004, pp. 2685-2696.

[21] L. Fang and W. Du, "A beacon-less location discovery scheme for wireless sensor networks," in In Proceedings of IEEE INFOCOM, 2005, pp. 13-17.

[22] L. Girod and D. Estrin, "Robust range estimation using acoustic and multimodal sensing," vol. 3, 2001.

[23] T. He, C. Huang, B. M. Blum, J. A. Stankovic, and T. Abdelzaher, "Range-free localization schemes for large scale sensor networks," in Proceedings of the 9th annual international conference on Mobile computing and networking, ser. MobiCom '03. New York, NY, USA : ACM, 2003, pp. 81-95.

[24] Jeffrey, "SpotON : An Indoor 3D Location Sensing Technology Based on RF Signal Strength

[25] L. Hu and D. Evans, "Localization for mobile sensor networks," in Proceedings of the 10th annual international conference on Mobile computing and networking, ser. MobiCom '04. New York, NY, USA : ACM, 2004, pp. 45-57.

[26] K. Langendoen and N. Reijers, "Distributed localization in wireless sensor networks : a quantitative comparison," Comput. Netw., vol. 43, no. 4, pp. 499-518, Nov. 2003.

[27] L. Lazos and R. Poovendran, "Serloc : Robust localization for wireless sensor networks," ACM Trans. Sen. Netw., vol. 1, no. 1, pp. 73-100, Aug. 2005.

[28] Isaac Amundson and Xenofon D. Koutsoukos. 2009. A survey on localization for mobile wireless sensor networks. In Proceedings of the 2nd international conference on Mobile entity localization and tracking in GPS-less environments (MELT'09), Richard Fuller and Xenofon D. Koutsoukos (Eds.). Springer-Verlag, Berlin, Heidelberg, 235-254.

[29] A. Nasipuri and K. Li, "A directionality based location discovery scheme for wireless sensor networks," in Proceedings of the 1st ACM international workshop on Wireless sensor networks and applications, ser. WSNA '02. New York, NY, USA: ACM, 2002, pp. 105-111.

[30] D. Niculescu and B. Nath, "Ad hoc positioning system (aps," in In GLOBECOM, 2001, pp. 2926-2931.
[31] _ _DV Based Positioning in Ad Hoc Networks," Telecommunication Systems, vol. 22, no. 1, pp. 267-280, Jan. 2003.

[32] A. Rao, S. Ratnasamy, C. Papadimitriou, S. Shenker, and I. Stoica, "Geographic routing without location information," in Proceedings of the 9th annual international conference on Mobile computing and networking, ser. MobiCom '03. New York, NY, USA: ACM, 2003, pp. 96-108.

[33] N. Sastry, U. Shankar, and D. Wagner, "Secure verification of location claims," in Proceedings of the 2nd ACM workshop on Wireless security, ser. WiSe '03. New York, NY, USA: ACM, 2003, pp. 1-10.

[34] C. Savarese, J. M. Rabaey, and K. Langendoen, "Robust positioning algorithms for distributed ad-hoc wireless sensor networks," in Proceedings of the General Track of the annual conference on USENIX Annual Technical Conference, ser. ATEC '02. Berkeley, CA, USA: USENIX Association, 2002, pp. 317-327.

[35] A. Savvides, H. Park, and M. B. Srivastava, "The n-hop multilateration primitive for node localization problems," Mob. Netw. Appl., vol. 8 , no. 4, pp. 443-451, Aug. 2003.

[36] Y. Shang, W. Ruml, Y. Zhang, and M. P. J. Fromherz, "Localization from mere connectivity," in Proceedings of the 4th ACM international symposium on Mobile ad hoc networking \& computing, ser. MobiHoc '03. New York, NY, USA; ACM, 2003, pp. 201-212.

[37] L. Doherty, K. S. J. Pister, and L. El Ghaoui, "Convex position estimation in wireless sensor networks," in INFOCOM 2001. Twentieth Annual Joint Conference of the IEEE Computer and Communications Societies. Proceedings. IEEE, vol. 3, 2001, pp. 1655-1663.

[38] Xuemin Shen, Jon W. Mark, and Jun Ye. 2002. Mobile Location Estimation in CDMA Cellular Networks by Using Fuzzy Logic. Wirel. Pers. Commun. 22, 1 (July 2002), 57-70. DOI=10.1023/A: 1016025802932 http://dx.doi.org/10.1023/A:1016025802932.

[39] R. Graham, "An efficient algorithm for determining the convex hull of a finite planar set," Information Processing Letters, pp. 132-133.

[40] R. A. Jarvis, "On the identification of the convex hull of a finite set of points in the plane," Inf. Process. Lett., vol. 2, no. 1, pp. 18-21, 1973.

[41] “About glomosim," http://pcl.cs.ucla.edu/projects/glomosim/, cited July 2011.

[42] “Omnet++ community site," http://www.omnetpp.org/, cited July 2011.

[43] “Opnet technologies," http://www.opnet.com/, cited July 2010.

[44] "The network simulator," http://www.isi.edu/nsnam/ns/, cited July 2010.

[45] M. Broxton, J. Lifton, and J. A. Paradiso, "Localization on the pushpin computing sensor network using spectral graph drawing and mesh relaxation," SIGMOBILE Mob. Comput. Commun. Rev., vol. 10, pp. 1-12, January 2006.

[46] A. A. Ahmed, H. Shi, and Y. Shang, "Sharp : A new approach to relative localization in wireless sensor networks," in Proceedings of the Second International Workshop on Wireless Ad Hoc Networking Volume 09, ser. ICDCSW '05. Washington, DC, USA: IEEE Computer Society, 2005, pp. 892-898.

[47] T. Camp, J. Boleng, and V. Davies, "A survey of mobility models for ad hoc network research," WIRELESS COMMUNICATIONS \& MOBILE COMPUTING (WCMC): SPECIAL ISSUE ON MOBILE AD HOC NETWORKING : RESEARCH, TRENDS AND APPLICATIONS, vol. 2, pp. 483-502, 2002. 